\title{
Técnica inadecuada en el empleo de inhaladores en pacientes atendidos en una consulta de neumología
}

\author{
R. GOLPE GÓMEZ, A. MATEOS COLINO, I. SOTO FRANCO
}

\section{Unidad de Neumología y Servicio de Medicina Interna. Hospital Comarcal de Monforte} de Lemos (Lugo)

\author{
INADEQUATE INHALERS TECHNIOUE IN PATIENTS ATTENDED \\ AT AN AMBULATORY RESPIRATORY WARD
}

\section{RESUMEN}

Objetivo: Evaluar la técnica de inhalación en enfermos ambulatorios tratados con inhaladores, atendidos en una consulta de neumología, e identificar factores asociados con un empleo inadecuado de los mismos.

Método: Se estudiaron prospectivamente 107 sujetos, de los cuales 100 completaron el estudio, determinando la técnica de empleo de inhaladores según listas de comprobación previamente elaboradas. Se buscaron diferencias significativas entre pacientes con técnica correcta e incorrecta.

Resultados: Edad media: $68 \pm 11$ años. 68 varones y 32 mujeres. Sólo el $31 \%$ de los pacientes realizaron una técnica de inhalación correcta cuando fueron evaluados por primera vez. Los pacientes que usaban cartuchos presurizados sin empleo de cámara espaciadora presentaron mayor número de errores que los que usaban otros dispositivos. El principal factor asociado con una mala técnica de inhalación fue la ausencia de una instrucción adecuada en el momento en que el tratamiento fue prescrito por primera vez. Los médicos generales ofrecieron esta instrucción con meds frecuencia que otros facultativos. No encontramos relaciones significativas entre una mala técnica de inhalación con la edad o sexo de los pacientes, o con el medio (hospitalario o ambulatorio) donde el tratamiento había sido prescrito.

Conclusiones: El empleo incorrecto de los inhaladores es un hallazgo frecuente, y una instrucción adecuada parece asociarse al mejor uso de los mismos. Es preciso sensibilizar al personal sanitario sobre la importancia de adiestrar a los pacientes en el empleo de los inhaladores como una parte de la atención integral de los mismos.

PALABRAS CLAVE: Inhaladores. Enfermedad pulmonar obstructiva crónica. Asma. Educación del paciente.

\section{ABSTRACT}

Objective: To assess inhalation technique in out-patients attending a respiratory ward who were using inhaler devices, and to identify factors associated with misuse of these therapies.

Method: One hundred and seven patients were prospectively studied, of whom 100 completed the study; Inhalation technique was evaluated using a previously elaborated check list. We searched for significant dif ferences between individuals with good or bad inhalation technique.

Results: Mean age: $68 \pm 11$ years. 68 males and 32 females. Only $31 \%$ of cases were observed to make no inhalation errors when first eva luated. Individuals using metered-dose inhalers without spacer chamber made significantly more mistakes than patients using other devices. The main factor associated with poor inhalation technique was found to be the absence of adequate supervised instruction when the treatment was first prescribed. General practitioners were less likely to offer this ins truction than other specialists. We did not find significant associations between inhaler misuse and patients' age, sex or the setting (hospital or ambulatory ward) where the treatment was first prescribed.

Conclusions: Incorrect use of inhalers is a frequent finding, and an adequate instruction seems to associate with improvement of patients' skills with use of these devices. Health personnel should be aware that inhalation technique instruction is essential ingredient of patients' management.

KEY WORDS: Inhalers. Lung diseases obstructive. Asthma. Patient edu cation.

Golpe Gómez R, Mateos Colino A, Soto Franco I. Técnica inadecuada en el empleo de inhaladores en pacientes atendidos en una consulta de neumología. An Med Interna (Madrid) 2001; 18: 69-73.

\section{INTRODUCCIÓN}

La administración de fármacos por vía inhalatoria constituye la base del tratamiento de los pacientes con enfermedad pulmonar obstructiva crónica (EPOC) y asma. Dada la elevada prevalencia de estas enfermedades $(1,2)$, la prescripción de estos fármacos constituye una parte significativa del gasto sanitario. Todos los sistemas de admi- nistración de los fármacos inhalados requieren un período de aprendizaje por parte del paciente, y es frecuente el empleo incorrecto de los mismos (3-5). El objetivo del presente trabajo es determinar el porcentaje de pacientes atendidos por primera vez en una consulta de neumología que emplean incorrectamente los inhaladores, y tratar de identificar factores asociados con el mal uso de los mismos.

Trabajo aceptado: 25 de Julio de 2000

Correspondencia: Rafael Golpe Gómez. Unidad de Neumología. Hospital Comarcal. C/ Corredoira s/n. - 27400 Monforte de Lemos (Lugo) 


\section{PACIENTES Y MÉTODO}

Se estudiaron prospectivamente 107 pacientes consecutivos de ambos sexos, remitidos a la consulta externa de neumología de nuestro centro (Hospital Comarcal, población de referencia: 60.000) con el diagnóstico de EPOC y asma. El criterio de inclusión era el empleo de medicación inhalada, mediante cualquier sistema comercial de administración, al ser atendidos por primera vez en la consulta. Se excluyeron a pacientes menores de 18 años o con alteraciones físicas o mentales graves que los incapacitasen manifiestamente para comprender el empleo de los inhaladores, o accionar los mismos.

Uno de los autores registró el número y tipo de inhaladores utilizado por el paciente, la especialidad del médico que lo había pautado, el lugar de la indicación (hospital o ambulatorio), e interrogó al paciente sobre si había recibido o no instrucción supervisada por parte de personal sanitario (médico o ATS) en el momento de la indicación. Se registró el tiempo transcurrido desde la indicación del tratamiento y la asistencia a la consulta.

Se realizó a todos los pacientes una espirometría forzada mediante un espirómetro de mano (Vitalograph 2120), siguiendo recomendaciones SEPAR (6). Se indicó al paciente que realizase las maniobras de inhalación de los fármacos, de la misma forma en que lo hacía habitualmente, utilizando para ello el/los sistemas de inhalación correspondientes, cargados con placebo. Se anotaron en la hoja de recogida de datos, el número y tipo de error cometido para cada sistema de inhalación, y el número total de errores por paciente. Se utilizaron unas listas de posibles errores elaboradas previamente, usando como guía las recomendaciones SEPAR para el empleo de este tipo de medicaciones (Tabla I) (7).

\section{TABLA I}

LISTA DE PO SIBLES ERRO RES EN EL EM PLEO DE LA M EDICACIÓN INHALADA, EM PLEADA EN EL PRESENTE ESTUDIO

Error tipo 1: $\quad$ No agitar o destapar el cartucho presurizado (M DI), o no cargar el dispositivo de polvo seco.

Error tipo 2: $\quad$ No introducir el inhalador o la cámara espaciadora en la boca.

Error tipo 3: $\quad$ No coordinar el disparo del M DI con la inspiración

Error tipo 4: $\quad$ No realizar una maniobra inhalatoria correcta para el dispositivo (lenta y profunda para el M DI, profunda y enérgica para los dispositivos de polvo seco).

\section{Error tipo 5: $\quad$ No aguantar la respiración unos 10} segundos tras inhalar el fármaco.

Error tipo 6: $\quad 0$ tros (especificar).

El médico modificó a continuación la prescripción de la medicación empleada, según lo consideró clínicamente indicado. Cuando la técnica demostrada por el paciente se consideró correcta (ningún error), no se modificó el sistema de administración, excepto en aquellos casos en que se prescribiese un principio activo no disponible comercialmente en el sistema de administración original. En el resto de los casos el médico indicó verbalmente al paciente los errores cometidos y le explicó la forma de corregirlos, demostrando a continuación él mismo la técnica correcta de administración con el placebo adecuado, e invitando al paciente a volver a realizar la prueba. Este procedimiento se repitió el número de veces preciso para que el paciente realizase la técnica de forma correcta o hasta que, a juicio del médico, quedó patente la incapacidad del paciente para utilizar el dispositivo de administración empleado. En este caso se realizó todo el proceso de nuevo con un sistema de inhalación diferente, hasta que el paciente consiguió una utilización correcta o, a juicio del médico, no mejorable.

Los pacientes fueron citados en consulta externa para una revisión en fecha posterior, y atendidos por el mismo médico, que volvió a revisar la técnica de inhalación empleada para cada dispositivo, según la metodología descrita más arriba, y anotó nuevamente los errores cometidos.

Para el análisis estadístico se empleó un programa para PCs (NCSS, Hintze JL, Kaysville, Utah). Se compararon los porcentajes mediante el test de Chi-cuadrado, y las medias mediante T-Student. Se usaron los correspondientes tests no paramétricos cuando los valores estudiados no seguían una distribución normal, evaluada ésta mediante el test de Kolmogorov-Smirnov. Se aceptó un valor de $\mathrm{p}<0,05$ como indicativo de significación estadística. Salvo que se indique otra cosa, los resultados se expresan como media \pm desviación estándar.

\section{RESULTADOS}

Se estudiaron 100 pacientes que concluyeron el estudio. Se excluyeron 4 pacientes que no acudieron a la segunda cita y 3 en los que se consideró que no estaba indicado tratamiento con medicación inhalada. Edad media: $68 \pm 11$ años (27-86). 68 eran varones y 32 , mujeres. 72 fueron diagnosticados de EPOC, y 28 de asma bronquial. El intervalo entre la primera y la segunda visita fue de $3,7 \pm 2,6$ meses.

La indicación de tratamiento había sido realizada por un médico general en 42 casos, un neumólogo en 36, un Internista en 10 , un médico perteneciente a un servicio de urgencias hospitalarias en 8 , un alergólogo en 2 , y un cardiólogo en 2 casos. El tratamiento se había indicado en un hospital en 30 casos y a nivel ambulatorio en 70 .

Los valores de la espirometría fueron los siguientes (se indica en paréntesis el valor porcentual respecto a los valores teóricos): FEV1: $1.338 \pm 646 \mathrm{ml} / \mathrm{s}(58 \pm 24 \%)$, FVC: $2.474 \pm 818$ $\mathrm{ml} / \mathrm{s}(82 \pm 20 \%)$, FEV1/FVC: $53 \pm 15 \%$. Cinco pacientes no colaboraron para la realización de espirometría.

Sólo 47 pacientes habían recibido instrucción supervisada sobre el empleo de los inhaladores al acudir por primera vez a consulta (en 40 casos realizada por un médico, y en 7 por un ATS). Los restantes 53 pacientes negaban haber recibido este tipo de instrucción.

La tabla II muestra el número de pacientes (y porcentaje respecto al total de cada subgrupo) que habían recibido instrucción supervisada, en relación con el lugar de indicación y la especialidad del médico que indicó el tratamiento. No existieron diferencias significativas respecto a si la indicación había sido realizada en el medio hospitalario o ambulatorio, pero sí respecto al médico que había sentado la indicación, siendo el porcentaje más bajo entre los generalistas. 
TABLA II

№ (PO RCENTAJE) DE PACIENTES Q UE HABÍAN RECIBIDO INSTRUCCIÓN SUPERVISADA SO BRE EL EM PLEO DE

INHALADO RES, A LA INDICACIÓ N DEL TRATAM IENTO

Según el lugar de indicación Según el médico que indicó el tratamiento

\begin{tabular}{ll}
\hline Ambulatorio: $30(43 \%)$ & Médico general: $10(24 \%)$ \\
Hospital: $17(56 \%)$ & Neumólogo: $24(66 \%)$ \\
& Internista: $7(70 \%)$ \\
& Alergólogo: $2(100 \%)$ \\
& Cardiólogo: $1(50 \%)$ \\
& Médico de urgencias: $3(37 \%)$ \\
& $p<0,01$ \\
\hline
\end{tabular}

La tabla III compara el tratamiento que traían pautado los enfermos al ser incluídos en el estudio con el que se indicó tras la primera visita a nuestro centro. No hubo diferencias en el número de dispositivos de inhalación prescritos por paciente. Sí las hubo, en cambio, en el tipo de inhalador empleado. El porcentaje de pacientes que usaban cartucho presurizado (MDI) directo (sin cámara espaciadora) disminuyó significativamente como resultado de la primera evaluación en nuestro centro, en relación con un empleo inadecuado y, a juicio del evaluador, no mejorable del mismo, mientras que aumentó la prescripción del MDI aplicado mediante cámara. Sin embargo, hay que precisar que seis pacientes en los que se indicó en la primera visita el empleo de MDI con cámara espaciadora, reconocieron haber abandonado el empleo de ésta al ser revisados en la segunda visita.

\section{TABLA III}

№ DE PACIENTES, CLASIFICADO S SEGÚN EL № DE DISPO SITIVOS EM PLEADOS PO R ENFERM O, Y EL TIPO DE INHALADO R UTILIZADO, A LA INTRODUCCIO N EN EL ESTUDIO (PRIMERA VISITA) Y EN LA SEGUNDA VISITA

\begin{tabular}{lccc}
\hline № de dispositivos & 1ㅁ visita & 2ª visita & P \\
\hline Uno & 78 & 82 & NS \\
Dos & 21 & 16 & NS \\
Tres & 1 & 2 & NS
\end{tabular}

Tipo de dispositvo

$M D$

MDI por cámara

Algún dispositivo de polvo seco

$\begin{array}{ccc}61 & 18 & <0,01 \\ 20 & 39 & <0,01 \\ 34 & 45 & \text { NS } \\ 1 & 43 & <0,01\end{array}$

Con respecto a los dispositivos de polvo seco, tras la primera visita no cambió significativamente el porcentaje de pacientes que usaban alguno de estos dispositivos. Sin embargo, cuando se inició el estudio, únicamente un paciente estaba tratado sólo con este tipo de dispositivos. En el resto de los casos, los dispositivos de polvo seco se combinaban con un MDI. Tras ser evaluados en nuestro centro, aumentó el número de enfermos tratados sólo con dispositivos de polvo seco.

Los errores más frecuentes en la primera visita fueron: no coordinar el disparo del MDI con la respiración (tipo 3), no realizar una maniobra inhalatoria correcta (tipo 4), y no aguantar la respiración tras la inhalación (tipo 5). El número de pacientes que cometieron cada tipo de error fue el siguiente: Tipo uno: 11, tipo dos: 4, tipo tres: 42 , tipo cuatro: 56 , tipo cinco: 63, tipo seis: 8 . En la segunda visita, los errores más habituales fueron asimismo los tipos 3, 4 y 5 .

Como "error tipo seis" se apreciaron los siguientes: repetir inmediatamente la dosis del medicamento inhalado, sin esperar unos 30 segundos, como es recomendable; exhalar a través del dispositivo de polvo seco antes de inhalar la dosis (con lo que, en teoría, se puede dispersar la dosis dispuesta para ser usada); y no acoplar el MDI a la cámara de inhalación (en este caso, el paciente introducía la boquilla de la cámara en la boca y disparaba el MDI colocándolo delante de la cámara, a distancia del orificio de la misma).

La tabla IV compara el porcentaje de pacientes que demostraban una técnica de inhalación correcta al ser incluídos en el estudio, y en la segunda visita. El porcentaje de pacientes que realizaron una técnica correcta, sin errores, aumentó significativamente en la segunda visita. Se muestra asimismo el porcentaje de pacientes con técnica correcta para los distintos dispositivos de inhalación, comparando la situación al ser incluídos en el estudio con los resultados en la segunda consulta. Aumentó significativamente el porcentaje de pacientes que empleaban correctamente el MDI y los dispositivos de polvo seco, considerados estos últimos en conjunto.

TABLA IV

PO RCENTAJES DE PACIENTES Q UE DEM UESTRAN UNA TÉCNICA CORRECTA EN EL EM PLEO DE INHALADO RES, COM PARANDO LA SITUACIÓN A LA INCLUSIÓN EN EL ESTUDIO (PRIM ERA VISITA) Y EN LA SEGUNDA VISITA

\begin{tabular}{lccc}
\hline & $\begin{array}{c}\text { Primera } \\
\text { visita }\end{array}$ & 2ª visita & $P$ \\
\hline Globalmente (todos los sistemas) & 31 & 66 & $<0,01$ \\
M DI directo & 18 & 66 & $<0,01$ \\
M DI por cámara & 50 & 51 & NS \\
Dispositivos de polvo seco (en conjunto) & 44 & 79 & $<0,01$ \\
\hline
\end{tabular}

Cuando se analizó si existía alguna diferencia en los porcentajes de pacientes que usaban correctamente un dispositivo concreto, en comparación con los otros dispositivos, se observó que, efectivamente, estas diferencias existían: El porcentaje de pacientes que usaban correctamente el MDI era claramente inferior al resto de los sistemas de inhalación. Esta diferencia se observó, con significación estadística, tanto al incluir al paciente en el estudio $(p=0,03)$ como en la segunda visita $(\mathrm{p}=0,04)$.

En la tabla V se analiza la relación de la edad, función respiratoria y tiempo previo que llevaban los pacientes emplean- 
do el tratamiento inhalatorio al ser incluídos en el estudio, con la realización o no de una técnica de inhalación correcta. No se encontraron diferencias en la edad o FEV1 entre ambos grupos. Inesperadamente, el tiempo medio de tratamiento antes de la inclusión en el estudio era significativamente inferior en los pacientes que realizaban una técnica correcta.

\section{TABLA V}

EDAD, FEV 1 (EN VALO RES PO RCENTUALES RESPECTO AL TEÓ RICO), Y TIEM PO PREVIO DE TRATAM IENTO CON LOS

INHALADORES, AL SER INCLUIDOS EN EL ESTUDIO, EN

PACIENTES CON TÉCNICA DE INHALACIÓN CORRECTA (CERO ERRO RES) E INCORRECTA

\begin{tabular}{lccc}
\hline & $\begin{array}{l}\text { Técnica } \\
\text { correcta }\end{array}$ & $\begin{array}{c}\text { Técnica } \\
\text { incorrecta }\end{array}$ & $\mathrm{P}$ \\
\hline Edad (años) & $65 \pm 12$ & $69 \pm 11$ & $\mathrm{NS}$ \\
FEV1 (\%) & $61 \pm 29$ & $56 \pm 11$ & $\mathrm{NS}$ \\
Tiempo de tto. (meses) & $28 \pm 51$ & $47 \pm 72$ & $\mathrm{P}=0,01$ \\
\hline
\end{tabular}

Finalmente, la tabla VI muestra la relación entre el empleo correcto de los inhaladores en el momento de inclusión en el estudio, y otros factores, como el sexo del paciente, la presencia o no de una instrucción supervisada a la indicación, el lugar donde se indicó el tratamiento, y el tipo de especialista que realizó la prescripción. Destaca la influencia de una instrucción adecuada en el empleo correcto de estos fármacos. Es preciso indicar que la asociación que existía aparentemente entre el tipo de especialista que realizó la indicación y el uso correcto de los inhaladores no se mantuvo con significación estadística cuando se corrigió para la realización o no por el médico en cuestión de una instrucción correcta, supervisada.

TABLA VI

№ (PO RCENTAJE) DE PACIENTES CON TÉCNICA DE INHALACIÓN CO RRECTA, SEGÚN DIVERSO S FACTO RES

\begin{tabular}{llcc}
\hline & & \multicolumn{3}{c}{$\begin{array}{c}\text { No (porcentaje) } \\
\text { de pacientes }\end{array}$} & P \\
\hline Sexo & Varones & $20(29 \%)$ & NS \\
Supervisión a la indicación & Mujeres & $11(34 \%)$ & \\
& Sí & $27(57 \%)$ & $<0,01$ \\
Lugar de indicación & No & $4(7 \%)$ & \\
Médico que realiza la & Ambulatorio & $13(43 \%)$ & NS \\
indicación $\left(^{*}\right)$ & Generalista & $5(25 \%)$ & \\
& Neumólogo & $17(47 \%)$ & $<0,01$ \\
& Internista & $6(60 \%)$ & \\
& M édico & & \\
& de urgencias & $2(25 \%)$ & \\
\hline
\end{tabular}

(*) Véase texto para más detalles.

\section{DISCUSIÓN}

Los resultados hallados son congruentes con otros trabajos, que han encontrado un elevado porcentaje de fallos técnicos en el empleo de la medicación inhalada por parte de los pacientes (4,8-11). El principal factor asociado a una técnica inadecuada que hemos identificado es la ausencia de una supervisión correcta al indicar el tratamiento. Cuando se corrige para este factor, no existen diferencias según el tipo de especialista que prescribe el tratamiento, resultado que se ha encontrado asimismo en otros estudios (11). Es de destacar, sin embargo, que el porcentaje de generalistas que, en nuestro trabajo, supervisaron adecuadamente la técnica de sus pacientes fue especialmente bajo, lo que posiblemente esté en relación con la sobrecarga asistencial de las consultas de atención primaria.

Hay que remarcar que otros estudios han demostrado un imperfecto conocimiento por parte del personal sanitario sobre el correcto empleo de estos tratamientos, lo que sin duda influye en la baja oferta de instrucción supervisada a los enfermos $(4,12-13)$.

El MDI empleado directamente, sin utilizar cámara espaciadora, fue el dispositivo que ocasionó más problemas para su uso correcto, lo que motivó su sustitución por un sistema alternativo en un elevado número de casos. Otros estudios confirman que este dispositivo es el más difícil de usar por un importante porcentaje de enfermos (14). Aparentemente, el ofrecer una instrucción supervisada, aunque reduce el porcentaje de enfermos que usan mal el MDI, no lo iguala a los otros inhaladores: en nuestro estudio, seguimos encontrando un mayor porcentaje de fallos con éste que con otros dispositivos al evaluar por segunda vez a nuestros pacientes, si bien la validez de esta observación es relativa, por las limitaciones de diseño del presente trabajo (ver más abajo).

Es importante notar que varios pacientes abandonan otros sistemas alternativos en favor del MDI, pese a explicárseles detalladamente sus errores. En nuestros pacientes, estos justificaron esta decisión en todos los casos porque la sensación producida por el impacto del chorro de gas en la orofaringe les produce la impresión subjetiva de que el medicamento es más eficaz.

Hemos observado asimismo que en muchas ocasiones se combina un dispositivo de polvo seco con un MDI, lo que podría, dada la distinta técnica de empleo de los mismos, contribuir a aumentar los errores de los pacientes. Tras ser evaluados en nuestra consulta, aumentó el número de pacientes tratados sólo con un dispositivo de polvo seco, aunque como se discute más abajo, de este trabajo no se puede deducir una influencia de esta estrategia en reducir el número de errores cometidos.

Al contrario de lo que habíamos esperado a priori, no encontramos relación entre la edad del paciente, o el medio (hospital o ambulatorio) en que se realiza la indicación, y la habilidad para emplear los inhaladores. La falta de una adecuada supervisión del empleo del tratamiento en el medio hospitalario (también en contra de lo que habíamos anticipado) explica probablemente el segundo de estos hallazgos, que contrasta con lo comunicado por otros autores (15).

Con respecto a la influencia de la edad, otros autores han comunicado resultados discordantes: mientras que algunos encuentran una mayor dificultad para el empleo de estos medicamentos en los pacientes de más edad (5), otros no encuentran una clara influencia de este factor (16). 
Tampoco encontramos una relación clara entre el sexo del paciente, o su función pulmonar, y el empleo de estos medicamentos de forma correcta. Sí hallamos, en cambio, relación entre el tiempo que el paciente llevaba tomando el tratamiento y su uso correcto, pero no en el sentido que esperábamos: Habíamos anticipado que los pacientes que llevasen más tiempo empleando la medicación la utilizarían de modo más correcto, mientras que nuestros resultados sugieren que se produce el fenómeno inverso. Quizás ello sea debido a que los pacientes van desarrollando con el tiempo más vicios que repercuten en la técnica.

El diseño del presente trabajo no permite analizar de forma correcta la influencia que la instrucción ofrecida en la consulta de neumología (o el cambio en el sistema de inhalación) pudiera haber tenido en los pacientes: aunque globalmente se redujo el número de errores, el hecho de que la misma persona evaluase al paciente en las dos visitas posibilita la existencia de sesgos dependientes del observador (17). Por otra parte, los pacientes incluidos en el estudio no pueden considerarse una muestra representativa de la población general, al haberse seleccionado sujetos remitidos a una consulta especializada, que presumiblemente son los que presentan un peor control clínico (lo que a su vez puede relacionarse con el empleo incorrecto de la medicación). No obstante esto no resta validez a la asociación entre la realización de una técnica de inhalación correcta y la oferta previa de una instrucción adecuada en este grupo de enfermos. Los estudios existentes en la literatura médica sobre población general son congruentes con nuestros hallazgos.

En conclusión, nuestro estudio muestra una elevada prevalencia de errores en el empleo de la medicación inhalada por parte de los pacientes remitidos a una consulta especializada, en nuestro medio. La oferta de esta instrucción por parte del personal sanitario parece inadecuadamente baja, por lo que es fundamental sensibilizar al mismo sobre la importancia de este tema.

Debe prestarse especial atención al empleo del MDI, cuya utilización se asocia a más errores que con otros sistemas de administración. Su sustitución por otros sistemas, o su asociación con una cámara de inhalación debe considerarse en los casos en que se observen dificultades con su empleo.

\section{Bibliografía}

1. Sobradillo V, Miravitlles M, Jiménez CA, Gabriel R, Viejo JL, Masa F, et al. Estudio IBERCOP en España: prevalencia de síntomas respiratorios habituales y de limitación crónica al flujo aéreo. Arch Bronconeumol 1999; 35: 159-66

2. Grupo Español del Estudio Europeo del Asma. Estudio Europeo del Asma. Prevalencia de hiperreactividad bronquial y asma en adultos jóvenes de cinco áreas españolas. Med Clin (Barc) 1996; 106: 761-77.

3. Crompton GK. The adult patient's difficulties with inhalers. Lung 1990; 16 Suppl: 658-62.

4. Plaza V, Sanchís J. Medical personnel and patient skill in the use of metered dose inhalers: a multicentric study. CESEA Group. Respiration 1998; 65: 195-8

5. Van Beerendonk I, Mesters I, Mudde AN, Tan TD. Assessment of the inhalation technique in outpatients with asthma or chronic obstructive pulmonary disease using a metered-dose inhaler or dry powder device. J Asthma 1998; 35: 273-9.

6. Sanchís Aldás J, Casan Clarà P, Castillo Gómez J, González Mangado N, Palenciano Ballesteros L, Roca Torrent J. Espirometría forzada. En: Recomendaciones SEPAR. Caminero Luna JA, Fernández Fau L (Eds.). Doyma S.A., Barcelona, 1998; 1-18.

7. Giner Donaire J, Basualdo Martín LV, Casan Clarà P, Hernández Carcereny C, Macián Gisbert V, Martínez Sanz I, Mengíbar Bellon A. Utilización de Fármacos inhalados. En Recomendaciones SEPAR. Caminero Luna JA, Fernández Fau L (Eds.). Doyma S.A., Barcelona, 1998; 399-420.

8. De Blaquiere P, Christensen DB, Carter WB, Martín TR. Use and misu-

se of metered-dose inhalers by patients with chronic lung disease. Am Rev Respir Dis 1989; 140: 910-6.

9. Erickson SR, Horton A, Kirking DM. Assessing metered-dose inhaler technique: comparison of observation vs. Patient self-report. J Asthma 1998; 35: 575-83.

10. Hilton $\mathrm{S}$. An audit of inhaler technique among asthma patients of 34 general practitioners. Br J Gen Pract 1990; 40: 505-6.

11. Larsen JS, Hahn M, Ekholm B, Wick KA. Evaluation of conventional press-and-breathe metered-dose inhaler technique in 501 patients. J Asthma 1994; 31: 193-9.

12. Jones JS, Holstege CP, Riekse R, White L, Bergquist T. Metered-dose inhalers: do emergency health care providers know what to teach. Ann Emerg Med 1995; 26: 308-11.

13. Guidry GG, Brown WD, Stogner SW, George RB. Incorrect use of metered dose inhalers by medical personnel. Chest 1992; 101: 31-3.

14. Van der Palen J, Klein JJ, Kerkhoff AH, van Herwaarden CL. Evaluation of the effectiveness of four different inhalers in patients with chronic obstructive pulmonary disease. Thorax 1995; 50:1183-7.

15. Allen SC, Prior A. What determines whether an elderly patient can use a metered dose inhaler correctly? Br J Dis Chest 1986; 80:45-9.

16. Gray SL, Williams DM, Pulliam CC, Sirgo MA, Bishop AL, Donohue JF. Characteristics predicting incorrect metered-dose inhaler technique in older subjects. Arch Intern Med 1996; 156: 984-8.

17. O'Connell MB, Hewitt JM, Lackner TE. Consistency of evaluators assessing inhaler technique. Ann Allergy 1991; 67:603-8. 